\title{
Large scale magnetic fields of our Galaxy
}

\author{
JinLin Han \\ National Astronomical Observatories, Chinese Academy of Sciences, Jia20, DaTun Road, \\ ChaoYang District, Beijing, China. email: hjl@nao.cas.cn
}

\begin{abstract}
Large-scale magnetic fields in the Galactic disk have been revealed by distributions of pulsar rotation measures (RMs) and Zeeman splitting data of masers in star formation regions, which have several reversals in arm and interarm regions. Magnetic fields in the Galactic halo are reflected by the antisymmetric sky distribution of RMs of extragalactic radio sources, which have azimuthal structure with reversed directions below and above the Galactic plane. Large-scale magnetic fields in the Galactic center probably have a poloidal and toroidal structure.
\end{abstract}

Keywords. ISM: magnetic fields, pulsars: general, Galaxy: structure

Magnetic fields of our Galaxy is fundamentally important in understanding the detected cosmic rays, star formation processes, and the origin of magnetic fields in the universe. Measuring the large-scale Galactic magnetic fields is the first and necessary step for further researches. Here I summarize the updated knowledge of large-scale magnetic fields in our Galaxy.

In the Galactic disk, the best probes for the magnetic fields are pulsars. RMs, together with dispersion measures, of pulsars near the tangential regions can directly and most efficiently to reveal the magnetic fields along the spiral arms where the line of sight is almost parallel to the large-scale fields. It has now been confirmed using the updated RM list of 1025 pulsars (Han et al. 2009) that magnetic fields in the spiral arms (i.e. the Norma arm, the Scutum and Crux arm, and the Sagittarius and Carina arm) are always counterclockwise in both the first and fourth quadrants, though occasionally some disordered fields appear in some segments of arms. Magnetic fields between these arms are always clockwise. Magnetic fields in arm regions and interarm regions show clear continuity in the fourth quadrant to the first quadrant. The arminterarm field reversals are very probably related to the streaming motions of the interstellar gas (Han et al. 2006). We noticed that the averaged variation of RMs of extragalactic radio sources along the Galactic longitudes (Brown et al. 2007) are consistent with the field reversal pattern obtained from pulsar RMs (see Han 2007 for comparison).

Supposing that the large-scale magnetic fields always go along arms, pulsar RMs become less efficient to probe the magnetic fields in the regions where the lines of sight to pulsars get more perpendicular to the spiral arms. Also, distant pulsars are very faint and hard to be observed for RMs, especially in the farther half of the Galactic disk. The distribution of the median magnetic fields of $\mathrm{OH}$ masers in HII and star formation regions can be used as supplementary tools for large-scale magnetic fields (Han \& Zhang 2007) in both the near and farther halves of the Galactic disk. Good perspective is justified by apparent coherent large-scale reversals in the distribution of magnetic fields from Zeeman splitting data of $\mathrm{OH}$ masers in a few $\mathrm{kpc}$ (see Fig. 1). More measurements of distant masers are needed to confirm this.

In the Galactic halo, large-scale magnetic fields have been qualitatively revealed by the antisymmetric RM sky of extragalactic radio sources in the inner Galaxy (Han et al. 1997 and 1999): Radio sources of $0^{\circ}<l<90^{\circ}$ mostly have positive RMs above the Galactic plane, but negative below the plane. Radio sources of $270^{\circ}<l<360^{\circ}$ mostly have positive RMs below the Galactic plane, and mostly negative above the plane. This is consistent with the large-scale toroidal fields in the halo with reversed directions above and below the Galactic plane. The antisymmetry has been recently confirmed by the RM distribution of much more radio sources (Taylor et al. 2009). However, the properties of the large-scale halo fields (e.g. field strength varies in what form and with what scale in height and radius) are not known yet.

In the Galactic center, within a few hundred pc, both poloidal and toroidal magnetic fields have been observed. See Han (2009) for a short summary and Ferriere (2009) for an extensive review, and please consult the references therein. The highly polarized non-thermal radio filaments within $1^{\circ}$ from the Galactic center indicate poloidal magnetic fields in the region. The 


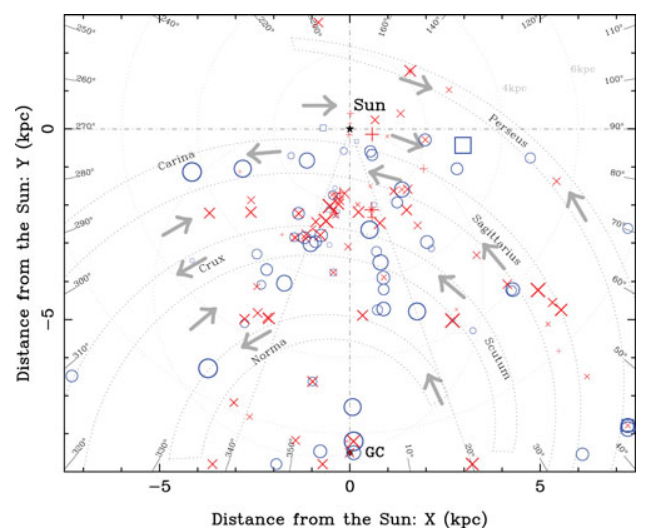

Figure 1. The distribution of the magnetic field median from Zeeman splitting measurements of $\mathrm{OH}$ masers (cross and circles) in 137 objects or HI or $\mathrm{OH}$ lines of 17 molecular clouds (plus and squares) projected onto the Galactic plane. Spiral arms and the magnetic field directions (arrows) derived from pulsar RM data are indicated. All crosses and pluses indicate the clockwise maser fields viewed from the Northern Galactic pole, and all circles and squares with counterclockwise fields. See Han \& Zhang (2007) for details.

filaments are probably illuminated flux tubes, with a field strength of about $1 \mathrm{mG}$. The diffuse radio emission of extent $400 \mathrm{pc}$ indicates for a weak pervasive field of tens of $\mu \mathrm{G}$. Polarized thermal dust emission detected in the molecular cloud zone at sub-mm wavelength is probably related to the toroidal fields parallel to the Galactic plane and complements the poloidal fields shown by the vertical filaments. It is possible that toroidal fields in the clouds are sheared from the poloidal fields, which explains the antisymmetric RM distribution of radio sources in this very central region. In a larger region, including the bulge, the large-scale magnetic fields should be closely related to the material structure, such as the bar. Observations for the magnetic fields there are very rare.

\section{Acknowledgements}

The author is supported by the National Natural Science Foundation (NNSF) of China (10821061, 10773016 and 10833003) and the National Key Basic Research Science Foundation of China (2007CB815403).

\section{References}

Brown, J. C., Haverkorn, M., Gaensler, B. M., et al. 2007, ApJ, 663, 258

Ferriere, K. 2009, A\&A, in press (arXiv:0908.2037)

Han, J. L. 2007, in: From Planets to Dark Energy: the Modern Radio Universe, Proceedings of Science (Published online at SISSA), Pos(MRU)072

Han, J. L. 2009, IAU Symp.259, p.455 (arXiv:0901.1165)

Han, J. L., Manchester, R. N., Berkhuijsen, E. M., \& Beck, R. 1997, AESA, 322, 98

Han, J. L., Manchester, R. N., \& Qiao, G. J. 1999, MNRAS, 306, 371

Han, J. L., Manchester, R. N., Lyne, A. G., Qiao, G. J., \& van Straten, W. 2006, ApJ, 642, 868

Han, J. L., van Straten, W., Manchester, R. N., \& Demorest, P. B. 2009, ApJ, to be submitted

Han, J. L. \& Zhang, J. S. 2007, A\&AA, 464, 609

Taylor, A. R., Stil, J. M., \& Sunstrum, C. 2009, ApJ, 702, 1230 